\title{
Fechamiento de Sedimientos en el Mar Caribe de Nicaragua, Bahia de bluefields, utilizando técnicas adicionales al análisis de Plomo-210
}

Victor Manuel Martinez

Centro para la Investigación en Recursos Acuáticos de Nicaragua, Universidad Nacional Autónoma de Nicaragua, Apdo. Postal 4598, Managua, Nicaragua. Correo electrónico: victor.martinez@cira-unan.edu.ni

\section{Recepcionado: 21 Diciembre 2009 / Aceptado: 26 Marzo 2010}

\section{Resumen}

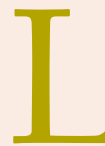

a Laguna de Bluefields es un ecosistema acuático somero e históricamente influenciado por actividades antropogénicas. Esta característica, hace a este cuerpo de agua poco apto para obtener un núcleo sedimentario idóneo para su fechamiento. Ante esta situación las técnicas nucleares, para definir sí un núcleo sedimentario es fechable o no, debe apoyarse en análisis secundarios.

En este estudio, los resultados de $\mathrm{Pb}-210$ se auxiliaron con determinaciones de Pérdidas por Ignición (PPI) y Porcentaje de Humedad en núcleos sedimentarios de dos sitios de la Laguna de Bluefields, para determinar la característica de fechamiento de los mismos. De acuerdo al análisis de humedad y $\mathrm{Pb}-210$, solo uno, de los dos núcleos sedimentarios, presentó algunas características de fechamiento.

Palabras claves: Técnicas nucleares, Mar Caribe de Nicaragua, Pérdida por Ignición, Porcentaje de Humedad.

\section{Abstract}

Bluefields lagoon is a shallow aquatic ecosystem and historically influenced by human activities. These particular conditions could cause limitations in dating the sediments with nuclear techniques. Secondary analysis has been carried out in combination with nuclear techniques to determine if sediment cores are datable in the Bluefields Lagoon. The secondary analysis of Loss on Ignition and percentage of humidity in sediment cores of two points in the Lagoon were employed. According to the analysis of humidity and dating with $\mathrm{Pb}-210$, only one of the two sediment cores presented some characteristics of dating. 


\section{Introducción}

La columna sedimentaria en un cuerpo de agua constituye un registro integral de los cambios temporales en aquellas áreas en que la acumulación de sedimentos tiene lugar sin bioturbación, -mezclado físico o episodios de erosión. Bajo estas condiciones, la columna sedimentaria registra información histórica de mucho valor sobre las características del ambiente en el momento de su formación, así como las modificaciones temporales producto de las actividades antropogénicas, (Golberg et al., 1963).

La Laguna de Bluefields es un ecosistema acuático somero y por razones históricas este ecosistema ha sido influenciado por actividades antropogénicas, desarrolladas por asentamientos humanos establecidos en su cuenca y en cuencas adyacentes. Es por eso que se hace necesario realizar una evaluación a priori de la característica de fechamiento de núcleos de sedimentos de este cuerpo de agua, que dada sus características, hace sospechar que la columna sedimentaria de este cuerpo de agua es poco apta para obtener perfiles de sedimentos que puedan ser fechados mediante el análisis de Plomo-210 y apoyado en la determinación de otros parámetros, tales como la Pérdida por Ignición (PPI) y el Porcentaje de Humedad. Los resultados de estos últimos parámetros nos dan una idea del grado de perturbación de los núcleos sedimentarios, es decir, el grado de mezcla, sedimentación y compactación de los mismos.

\section{Materiales y Métodos}

La captación de los núcleos sedimentarios se realizó en mayo del 2008; en dos sitios en el lóbulo sur de la laguna de Bluefields (figura 1); la selección de estos sitios se hizo siguiendo las recomendaciones del Manual del Proyecto Regional RLA/7/012/ (IAEA, 2009), las cuales se mencionan a continuación:
- Realizar el muestreo preferentemente en época seca

- Elegir sitios de depósito preferencial de sedimentos finos

- Seleccionar sitios que presenten tasas de sedimentación entre 0,3 y $1,0 \mathrm{~cm} \cdot a n ̃ o^{-1}$

- Evitar sitios con alta densidad de biota bentónica para precaver bioturbaciones en los perfiles de sedimentos

- Prevenir sitios en los cuales se realicen actividades de dragados

- Prescindir de sitios en los cuales se realicen actividades de pesca de arrastre

- Evitar sitios de alto tránsito de embarcaciones impulsadas con varas en el fondo

Las columnas de agua en los sitios 6 (Coordenada: Este 201012, Norte 1314817) y 7 (Coordenada: Este 195948, Norte 13107861), tienen una profundidad de 1.5 y 0.9 m respectivamente. Los núcleos sedimentarios fueron seccionados centímetro a centímetro y se les determinó el contenido de $\mathrm{Pb}$ 210, a la par se realizaron análisis que se utilizan

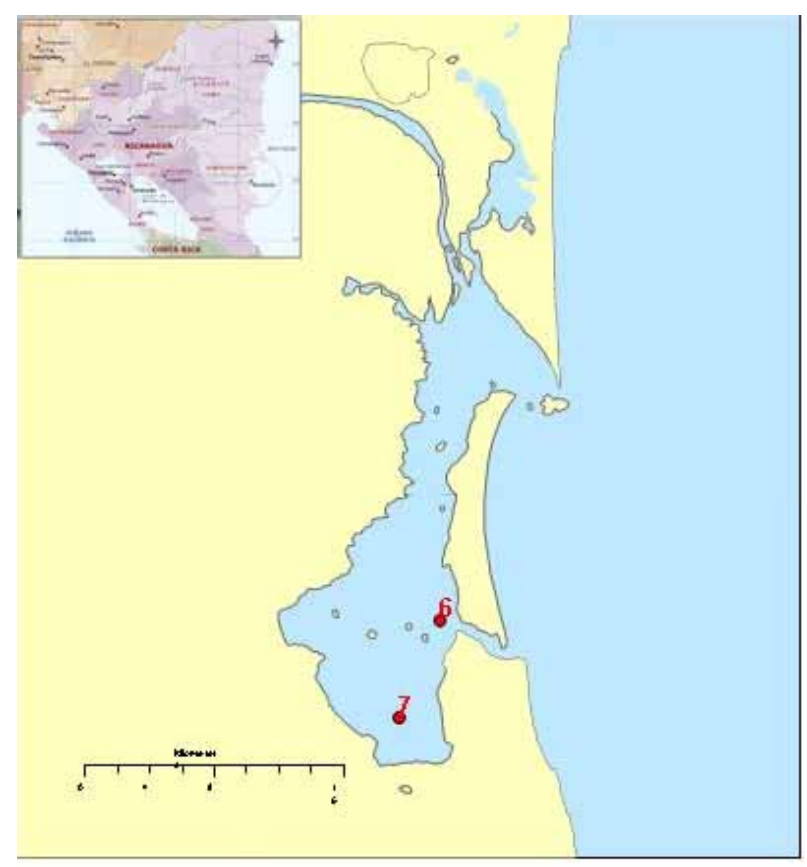

Figura 1. Sitios muestreados. Laguna de Bluefields, Nicaragua 
como herramientas complementarias para definir la idoneidad de algún perfil de sedimento para ser fechado; estos análisis son Pérdida por Ignición (PPI) y Porcentaje de Humedad.

\section{Resultados y Discusión}

De acuerdo a las profundidades de la Laguna de Bluefields, las cuales varían entre 0.9 y $3.6 \mathrm{~m}$, ésta es demasiada somera, por lo que se consideraría un cuerpo de agua poco apto para obtener un perfil de sedimento con buenas características para ser fechado.

\section{Análisis para seleccionar el perfil de sedimento más idóneo para fechar}

\section{Perfil de sedimento del sitio 6}

\section{Porcentaje de Humedad y Pérdidas por Ignición}

De acuerdo a los resultados que se describen en la figura 2, este perfil muestra una disminución poco significativa del contenido de agua a partir de la sección ubicada a los $39 \mathrm{~cm}$ de profundidad, a partir de esa profundidad hay indicios de la existencia de poca mezcla, sedimentación y poca compactación. Según esto, el perfil podría ser fechable.

Las pérdidas por ignición para el perfil captado en el sitio 6 (figura 2), muestran un aumento de los niveles de materia orgánica con la profundidad. Esto evidencia que el perfil podría estar mezclado en su capa superficial. En este perfil hay poca homogeneidad y en las capas inferiores de éste se alcanzan los valores más altos y heterogéneos de PPI, lo cual podría indicar una sedimentación no constante y que la degradación de la materia orgánica ha sido incompleta en las secciones más profundas.

De acuerdo a los resultados de los análisis de PPI en el perfil de sedimentos del sitio 6, no se puede determinar si este núcleo sedimentario es fechable o no.

Por el contrario, los resultados de la porosidad muestran una disminución con la profundidad, indicando aumento de la compactación de las secciones más profundas, indicando la presencia de valores bases que podrían reflejarse también en los resultados de $\mathrm{Pb}-210$, dando lugar a una posibilidad de fechar este perfil.
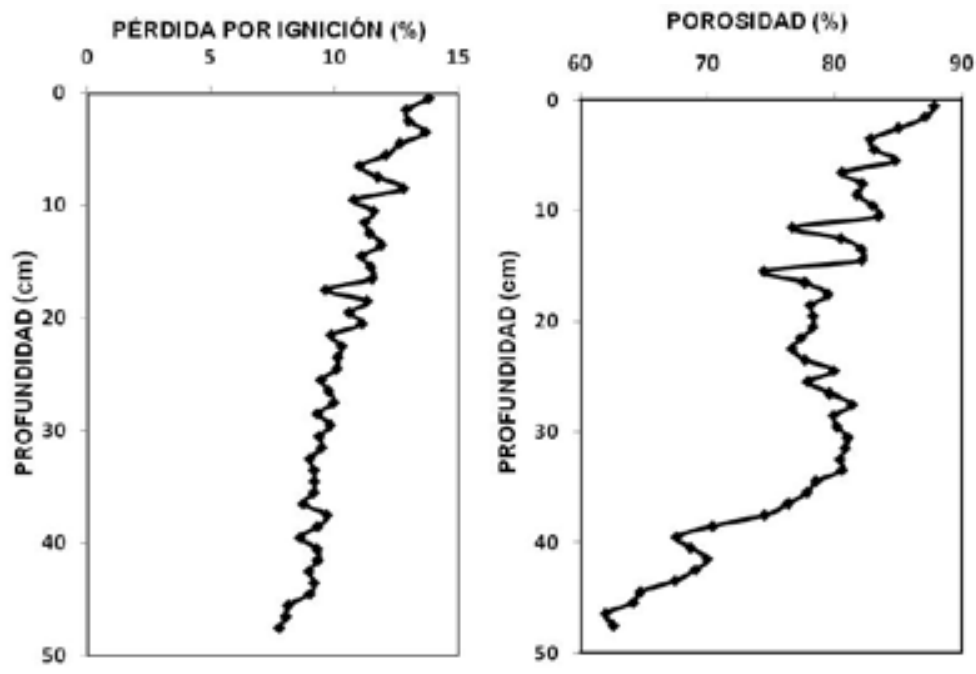

Figura 2. Relación entre PPI, Porosidad y Profundidad del perfil de sedimento del sitio 6 


\section{Perfil de sedimento del sitio 7}

\section{Porcentaje de Humedad y Pérdidas por Ignición}

El perfil de sedimento del sitio 7, muestra que hay poca homogeneidad (figura 3), lo cual podría indicar una mezcla en el perfil de sedimento, esto con respecto a las pérdidas por ignición. Además podría decirse que este perfil no alcanzó la profundidad debida; esto nos lleva a la conclusión que esta relación no podría ser tomada como una referencia para definir, sí este perfil es fechable o no.

Además este perfil de sedimento muestra un contenido de agua un poco homogéneo y en las últimas secciones este contenido tiende a aumentar; lo cual es una evidencia de mezcla, sedimentación y menos compactación. Esto indica que posiblemente este perfil no llegó a profundidad y por tanto, se le daría poco crédito para ser fechable.
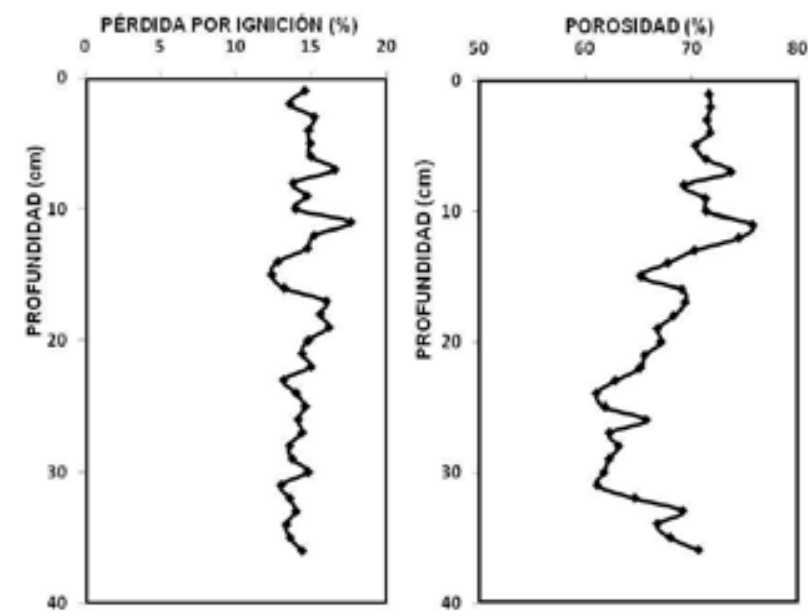

Figura 3. Relación entre PPI, Porosidad y Profundidad del perfil de sedimento del sitio 7

\section{Plomo-210 en los Perfiles de sedimentos}

\section{Perfil de sedimento del sitio 6}

Para este perfil los resultados que se presentan en la figura 4 corresponden a la relación de la actividad de $\mathrm{Pb}-210$ en función de la profundidad. En este gráfico se exceptúa el valor correspondiente a la sección 43; ya que resultó un valor aberrante de $341,8 \pm 23 \mathrm{Bk} /$ $\mathrm{kg}$. Según este perfil se observa un decrecimiento de la actividad de $\mathrm{Pb}-210$, aunque hay algunas zonas en las cuales se da un aumento. La presencia de estratificaciones en el núcleo sedimentario del sitio 6, demanda de un análisis más en detalles que podría dar lugar a realizar el fechamiento por tramos. Para definir, sí es fechable este perfil de sedimento se necesitaría conocer el $\mathrm{Pb}-210$ base para determinar el $\mathrm{Pb}-210$ en exceso y realizar la relación del $\ln \mathrm{Pb}-$ 210 exc y la masa acumulada, (Appleby, P.1994).

\section{Perfil de sedimento de estación 7}

En los primeros 15 centímetros del perfil de sedimento del sitio 7 (figura 4), se observa un buen decaimiento de la actividad del $\mathrm{Pb}-210$; manteniéndose casi constante a partir de esa sección. Sin embargo, no tenemos la certeza de que este perfil llegó al fondo, es por eso que el fechamiento de este perfil no sería muy confiable, (Barri, P. M. 1995).

Por lo anterior, podría decirse que una columna sedimentaria apta para ser fechable constituye una útil herramienta de monitoreo ambiental y que registros pueden proveer datos específicos acerca de los flujos de los contaminantes procedentes

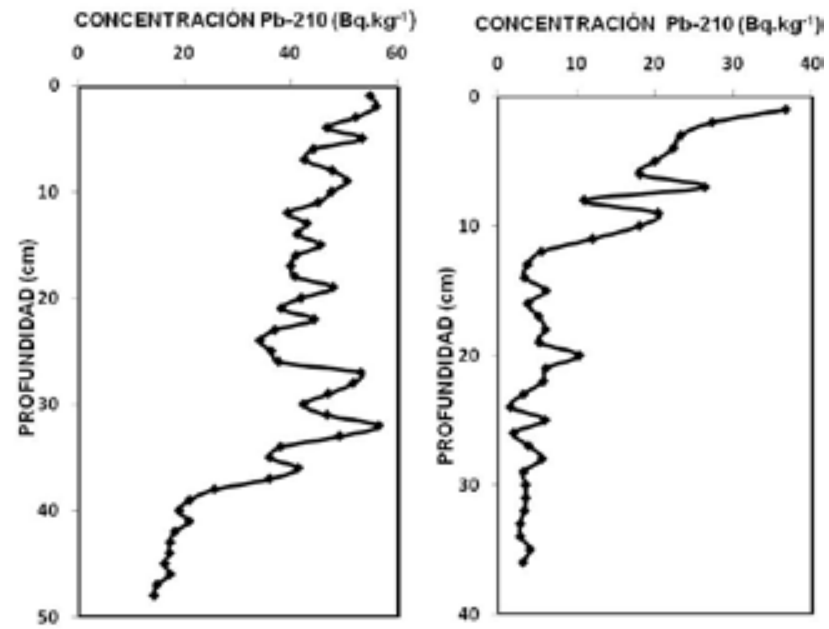

Figura 4. Relación entre $\mathrm{Pb}-210$ y la Profundidad de los perfiles de sedimento de los sitios 6 (gráfica a la izquierda) y 7 (gráfico a la derecha) 
del desarrollo urbano e industrial, (Axelsson et al., 1989; Valette-Silver, N. 1993), así como también información sobre las tasas de sedimentación cronológica, (Dumailo, S. 2003).

De acuerdo al contenido de humedad de los perfiles de sedimento, el único punto fechable correspondería al del sitio 6. Sin embargo, respecto a los resultados de PPI este perfil, al igual que el del sitio 7, no presentó una estratificación muy buena para tomarlo como referencia para ser fechado. Adicionalmente, el perfil del punto 7 , se quedó muy corto en profundidad.

\section{Conclusiones}

La laguna de Bluefields constituye un cuerpo de agua muy somero, característica que no la hace tan apta para obtener un buen perfil de sedimento para ser fechado. No obstante, de los dos núcleos sedimentarios analizados, el único perfil con posibilidades de ser fechado según el comportamiento de la actividad de $\mathrm{Pb}-210$, en función de la profundidad, es el punto 6.

\section{Agradecimientos}

Al Organismo Internacional de Energía Atómica por el apoyo financiero.

Al personal del CIRA/UNAN, en especial al del laboratorio de Radioquímica Ambiental, que contribuyó a la realización de los análisis de este estudio.

\section{Bibliografía}

Appleby, P. (1994). The ${ }^{210} \mathrm{~Pb}$ dating methodology, with emphasis on problems and solutions.

Axelsso, V., El-Daoushy. Sedimentation in the Edsviken bay studied by the $\mathrm{X}$-ray and the $\mathrm{Pb}-210$ methods. Geografiska Annale $71 A$.

Barri, P.M.(1995). Introducción a la datación de sedimentos marinos mediante la técnica del ${ }^{210} \mathrm{~Pb}$. Treball de Recerca.

Dumailo S. (2003). Evaluación de la problemática ambiental por medio del estudio de algunos aspectos de sedimentación y contaminación en la laguna de Bluefields, RAAS, Nicaragua. Tesis de Maestría, CIRA/ UNAN.

Goldberg E.D. (1963). Geochronology with ${ }^{210} \mathrm{~Pb}$, In: Radioactive Dating. Proceedings of a Symposium, International Atomic Energy Agency, Vienna., 121-131.

IAEA, (2009). Aplicación de técnicas nucleares en la solución de problemas específicos del manejo integrados de zonas costeras en el Gran Caribe. Manual del Proyecto Regional RLA/7/012/.

Valette-Silver N. (1993). The use of sediment cores to reconstruct historical trends in contamination of estuarine and coastal sediments. Estuaries.16:577-588. 